\title{
Overseas Legislation of Traditional Chinese Medicine : Problems and Countermeasures
}

\author{
Diangang Liu \\ School of Foreign Languages \\ Hubei University of TCM \\ Wuhan City, Hubei Province, 430065, PRC \\ liudiangang@163.com \\ Chi Gu \\ School of Foreign Languages \\ Hubei University of TCM \\ Wuhan City, Hubei Province, 430065, PRC \\ guchihbtcm@163.com
}

\author{
Herong Mao* \\ School of Foreign Languages \\ Hubei University of TCM \\ Wuhan City, Hubei Province, 430065, PRC \\ trans_morning123@126.com \\ Na Song \\ School of Foreign Languages \\ Hubei University of TCM \\ Wuhan City, Hubei Province, 430065, PRC
}

\begin{abstract}
As TCM further goes globally in the recent decades the overseas legislation of TCM has become a hot spot in the TCM-related rese arch. Through a systemic study, we have found a sequence of problems during the course of TCM legislation overseas, including high standard of language for TCM practitioners, tight restrictions on the use of Chinese medicinal herbs, limited coverage of TCM in the overseas medical insurance systems, etc. Relatively, we firmly believe that more practitioner-friendly language policies should be formed in the future, the development of Chinese medicinal herbs overseas should be promoted, and medical communication for improving the Recognition of TCM should be carried out urgently.
\end{abstract}

Keywords_overseas legislation; traditional Chinese medicine; problems; countermeasures

\section{INTRODUCTION}

According to Traditional Chinese Medicine in China issued by the State Council Information Office of the People's Republic of China in December 2016, [1]Traditional Chinese Medicine (TCM) has been increasingly spread overseas and more widely accepted in the world. The details are as follows: it has been spread to 183 countries and regions around the world, 103 member states under the framework of the World Health Organization have given approval to the practice of acupuncture and moxibustion, 29 have enacted special statutes on traditional medicine, and 18 have included acupuncture and moxibustion treatment in their medical insurance provisions, traditional Chinese herbal medicines have gradually entered the international system of medicines, and some of them have been registered in Russia, Cuba, Vietnam, Singapore, United Arab Emirates, and other nations, some 30 countries and regions have opened a couple of hundred TCM schools to train native TCM workers.

However, TCM legislations in some countries are far from sound because of the difference of medical laws in each country, different medical management systems and cultural backgrounds. For instance, the different states of America

This research is financially supported by academic programs of Hubei provincial department of education (2014318, 15Q112) have only passed the acupuncture-related laws, while in Switzerland there are only federal vocational exam plans which recognize four kinds of TCM diplomas. Because of discrimination against TCM or some other causes, there are quite a few countries which still do not have any legislative actions or plans until now, like Holland, Portugal, Laos, Cambodia, the Philippines and Malaysia etc.

\section{Problems Faced With the Overseas Legislation of TCM}

Although some countries have set a good example for the establishment of TCM-related rules and regulations overseas, many problems still exist in different countries in terms of TCM legislation.

\section{A. High Standard of Language for TCM Practitioners}

For the TCM practitioners in the western countries, most do well in their professional fields. However, they might have some problems in the use of foreign languages. Due to the cultural difference, the native language of the practitioners is usually not English or other local languages. However, during their medical practice, the practitioners need to communicate with the patients effectively. The demand of language for TCM practitioners has become a serious problem for TCM legislation in many countries.

For example, the applicants should have diploma of higher education in TCM and master the special language while applying for the license of TCM in Hungary. In Switzerland, the applicants must use the local language to take part in the exams to get a medical diploma about the Chinese medicine and acupuncture. According to the regulations of New Zealand government, the applicants who have no native diploma of medicine must take part in the local examination. The language used in the examination is of course English. The language problems have made many TCM practitioners, especially the senior and experienced practitioners difficult to get the legal certifications. 


\section{B. Tight Restrictions on the Use of Chinese Medicinal Herbs}

The quality and efficacy of Chinese medicinal herbs are always doubted and argued by foreigners. This is one of the

By the end of 2014, 1438 traditional herbal medicinal products including Chinese medicinal were registered with European traditional medicines. [2]Although great progress has been made during the last decade, many countries are putting forward tighter restrictions on the use of Chinese medicinal herbs in the recent years.

Many media and research groups have reported that they find toxic compounds or microorganisms in the tested Chinese medicinal herbal products. To these reports, we should also keep doubts about them. [3]There are examples of taking toxic materials into medicine in China. In the precious Chinese medicinal product Angong Niuhuang Wan, there are realgar and cinnabar, which follows the rule of Mutual Suppression in combination of Chinese medicinal. However, this curing method is unacceptable for foreigners.

\section{Limited TCM Coverage in the Overseas Medical Insurance Systems}

Generally speaking, the medical insurance system means that one country adopts the medical insurance benefits to pay for medicine and hospital bills for the masses. Many countries have adopted their own modes of medical insurance to manage and control the heath care expenses. Theoretically, TCM in other countries is also a treatment which should fall into the range of medical insurance, but often it is not the case. Taking Germany for example, the coverage of medical insurance does not include the expense of acupuncture service, which should be paid by the patients themselves according to the present German medical laws. Typically, TCM-related treatment cost is not small overseas. In this sense, such medical practices severely increase the economic burden of patients and force them to give up a try of TCM. Another example for Australia, due to the acupuncture is not included in universal health insurance coverage; so many people don't choose acupuncture to treat themselves. [4]

\section{COUNTERMEASURES TAKEN IN THE OVERSEAS LEGISLATION OF TCM}

\section{A. Formulating More Practitioner-friendly Language Policies}

Why some overseas TCM legislations impose strict language requirements? The key lies in the actual needs. The legislative bodies in some countries tend to take it for granted that the higher proficiency of the local languages that TCM doctors have, the more effective communication will be done between them and the patients during the process of diagnosing and treating the illnesses. But it is too difficult to master the second language for the aged doctors of TCM whose mother language is often Chinese and have spoken Chinese most of their lives. There is often a huge gap between their language ability and the requirement. In the light of such a situation, the legislative bodies in different countries should make an all-round research and take into consideration of the specificity of TCM industry in the local society. The main reasons why TCM is difficult to get legal status in a lot of countries. On April 30, 2004, the Europe began to enforce the EU Directive on Traditional Herbal Medicinal Products.

government should formulate strict but flexible language policies for different groups of the applicants. Only in this way can TCM doctors be promoted and encouraged to go in for a TCM career. Meanwhile, the TCM industry associations should face problems and come up with the ways of improving TCM doctors' language ability by giving lectures and classes in the required language etc. [5]

\section{B. Promoting the Development of Chinese Medicinal Herbs Overseas}

The development and regulation of any industry are interdependent on each other. The better one industry develops, the more urgent regulations and legislations are to keep up with the times. [6]The overseas development and legislation of Chinese medicinal herbs also abides by such a rule.

To make Chinese medicinal herbs legitimate to be registered in the overseas target market, the first and best way is to lead their development by the holistic development of TCM. In the short run, some TCM doctors may be eliminated from the medical market for they are less qualified with the sound development of TCM legislations over the world. This will affect the sales of Chinese medicinal herbs in some degree too. But in the long run, the strict TCM legislations will promote the development of Chinese medicinal herbs and increase their sales as more and more foreigners find it reliable and trustworthy to use them. In return, the mainstream society will know better about TCM and push forward healthier legislations on TCM.

\section{Enhancing Medical Communication to Improve the Recognition of TCM}

The main reason why the overseas mainstream society do not accept and recognize the effect of TCM is their failure to bring TCM into the range of medical insurance systems. We must enhance the cultural communication and popularize TCM vigorously overseas and change the local people's attitudes and recognition towards TCM to improve its social status.

On the one hand, we can disseminate the excellent skills and miraculous curing effect of TCM with the massive media campaign by inviting medical experts to communicate with the foreign masses to change the unsatisfying images of TCM in their eyes. On the other hand, for the misunderstandings and even the false conceptions of TCM among the foreigners, especially among the westerners, explanation and propaganda work should be done as immediately as possible. In order to achieve this goal, Chinese government as well as Chinese people can turn to modern platforms for communication, the TV and radio programs, the Internet, Wechat, twitter, just to mention a few. By enhancing the medical communication through modern means, TCM will be more widely recognized and accepted in the world. 


\section{CONCLUSION}

In conclusion, the overseas legislation of TCM has been through a couple of decades, during which a lot of progress has been made under the joint efforts of Chinese government, the local governments and TCM expert groups and practitioners.

Two rules can be concluded through the research of TCM legislative texts in the world. Apparently, legislation of TCM in many overseas countries had begun quite early, but the national conditions, regional disparity etc. lead to the reality that TCM develops rather slowly in these countries, such as India and the United Arab Emirates. On the contrary, although TCM legislation in some overseas countries started late, but the development of TCM is rather rapid, such as Nepal, Israel and Burma. There exists one similarity among these countries, that is, they are geographically contiguous to China. Culturally, they have been deeply affected by China's Confucianism, so they have similar cultural backgrounds. Because of the cultural closeness and economic complementarily, they are firm proponents and respondents of and China's 'Belt and Road' Initiative all the time. So these countries' recognition and acceptance levels of TCM are generally higher. For example, the Sino-Nepal United Hospital was established in the capital city Katmandu in 1992, which is a general hospital making use of western medicine and Chinese acupuncture, cupping and patent medicine. In 2006, the first Research Center for TCM was established in Nepal, which combines TCM research and development, clinical treatment with personnel training. As the Nepalese Health Minister once said, TCM has not merely cured diseases of Nepalese, promoted the development of friendship between Chinese and Nepalese people, but also carried forward TCM culture and solved the employment problem of the local people.[7]

However, many central and eastern European countries such as Belgium, Switzerland, and Czech Republic also follow the second law. Although they are far away from China with quite different cultures, TCM has become in recent years increasingly popular for its unique strengths in treating chronic diseases, obesity, pains etc. Since the first Health Ministers Meeting between China and CEEC held in 2014, China is forming closer ties with CEEC countries in the field of health, where the governmental cooperation in the field of TCM is a prominent part. The TCM Center then established in Czech Republic is dubbed the first center of TCM in the central and eastern European countries, which is also a milestone in the development of TCM in the western countries.

\section{ACKNOWLEDGMENTS}

This research is financially supported by academic programs of Hubei provincial department of education (2014318, 15Q112). Sincere thanks also go to colleagues from the Center for Compilation, Translation and International Communication of Jingchu TCM Literatures for their constructive suggestions in the writing of the paper.

\section{REFERENCES}

[1] China's State Council Information Office, Traditional Chinese Medicine in China, http://www.china.org.cn/chinese/201701/17/content_401209 42.htm,2017-01-17. (In Chinese)

[2] Chen Chen, An Overseas Chinese Dong Zhilin Talks about the Current Situation of TCM Development Internationally, http://www.worldtcm. org/160605/M05213448.shtml,2016-06-05.( In Chinese)

[3] Mason. S, P. Tovey, et., Evaluating Complementary Medicine: Methodological Challenges of Randomized Controlled Trials, London: British Medical Journal, 2002, vol.325, p.832.

[4] Yang Cuiying, Guo Guangzhi. Australian Social Security System, in Chinese, Shanghai: Shanghai People's Publishing House, 2009, p.149.( In Chinese)

[5] Chinese Medicine Board of Australia, http://www. chinesemedicineboard.gov.au/Codes- Guidelines/FAQ.aspx, 2012-07-01.

[6] The Regulation of Traditional Chinese Medicine-Australia and Overseas, http://www.health.vic.gov.au/archive/archive2006/chinese/report/9.htm\# 9.6. 2004.

[7] Liu Jinsheng, Hou Zeming, TCM in the World, in Chinese, Beijing: Beijing Science and Technology Publishing House, 2009, p.77. (In Chinese) 